\title{
Extended Breast Implant-Associated Anaplastic Large Cell Lymphoma: case report and discussion
}

\author{
Olivia Stas ${ }^{a}$, Elisabeth Mourin ${ }^{a}$, Julien Depaus ${ }^{a}$, François-Xavier Hanin ${ }^{b}$, Ivan Theate ${ }^{c}$, Marc André ${ }^{a}$ \\ a Université catholique de Louvain, CHU UCL Namur, Department of Hematology, Yvoir, Belgium; ${ }^{b}$ Université catholique de Louvain, CHU UCL Namur, \\ Department of Nuclear Medecine, Yvoir, Belgium; 'c.Institut de Pathologie et de Génétique (IPG), Department of Pathology, Gosselies, Belgium.
}

We report the case of a 69-year-old woman who presented an aggressive breast implant-associated anaplastic large cell lymphoma (BIA-ALCL) with supra and infra-diaphragmatic disease. The diagnosis was made 17 years after her first prosthesis, following a right breast carcinoma, and three years after the replacement of this first prosthesis.

BIA-ALCL is a rare form of non-Hodgkin lymphoma due to breast implant.

Unique features of this case include the fast clinical extension of a lymphoma that is indolent in the vast majority of the cases. Indeed, less than 2 months after the first breast symptoms, cutaneous metastasis appeared on the right arm (figure 1, figure 2).
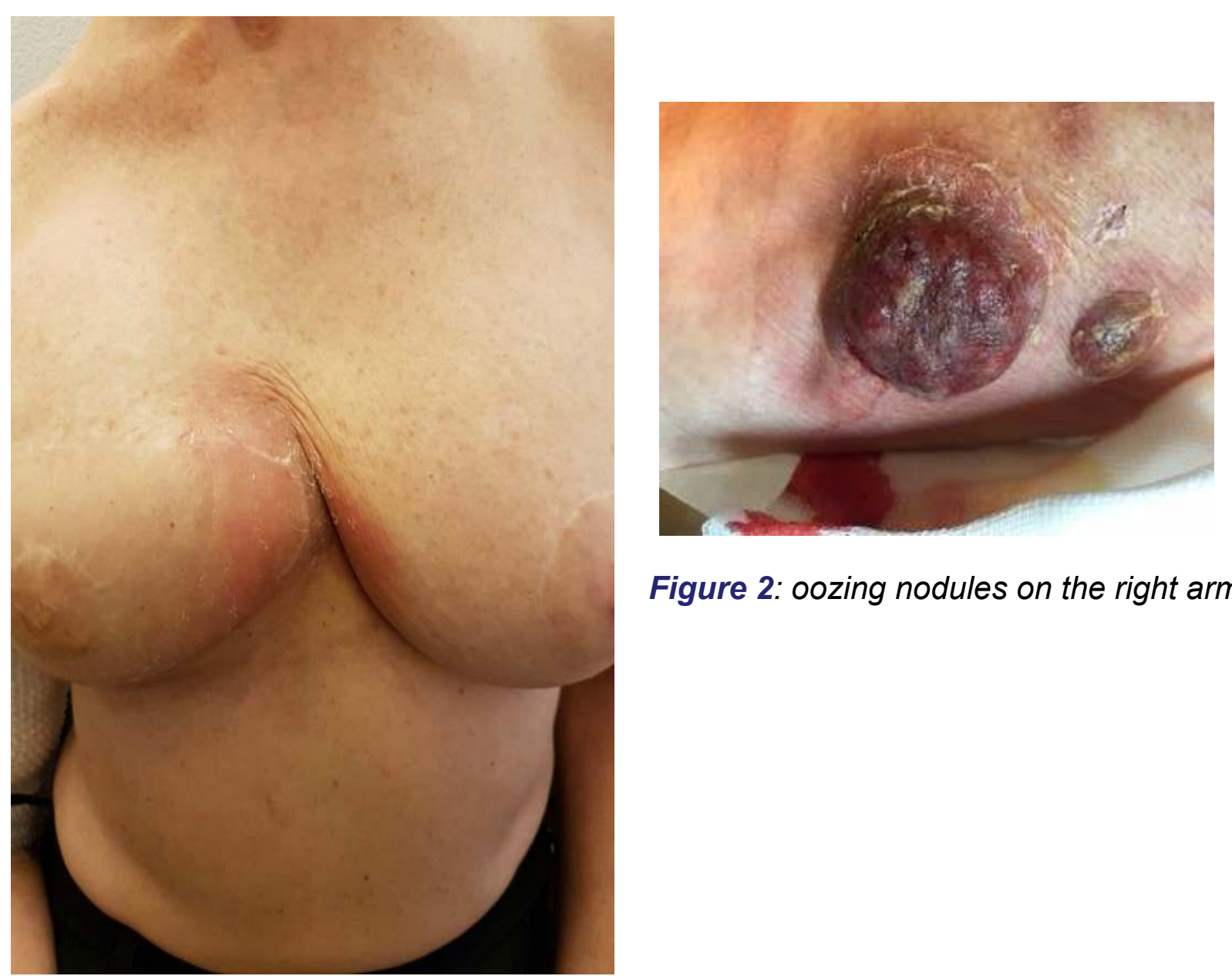

Figure 2: oozing nodules on the right arm

Figure 1: redness on the internal side of the right breast, on inter-mammary and the infero-medial quadrant of the left breast.

The key diagnosis exams are histology and immunohistochemistry including CD30 and cytotoxic markers, and PET-scan to evaluate the extension of the disease. The PET-scan showed mammary right tumor extended on the right arm, with digestive and inferior left leg extension (figure 3). The treatment should include removal of the prosthesis and any associated mass. Local residual or unresectable disease may benefit from radiation therapy to the chest wall. For regional lymph node involvement or confirmed extended disease, adjuvant chemotherapy more in line with systemic ALCL ALKnegative treatments is recommended.
Finally, brentuximab vedotin, an anti-CD30 monoclonal antibody, showed encouraging results in refractory disease but still need more prospective trials.

Some studies have been conducted to explain the pathogenesis and risk factors of BIA-ALCL that can appear for either cosmetic or reconstructive indications. One of them explained the implication of chronic T-cell stimulation against the prosthetic material, with local antigenic drive, leading to genetic degeneration, dysplasia and ultimately to lymphoma.

Another study compared capsules of textured and smooth implants in pigs and showed that there are increased lymphocytes on textured breast implants, with a T-cell predominance. In a study comparing implant capsules between patients with BIA-ALCL and patients with normal capsular contracture, investigators underscored a high bacterial count and the predominance of a gram-negative bacteria Ralstonia pickettii in BIA-ALCL patients, which is a common contaminant of drinking water and soils. This study confirms the evidence that standard care of sterility must be respected by every surgery center involved in placing breast implant and that further research is required for identification of risk factors and prevention of future cases.

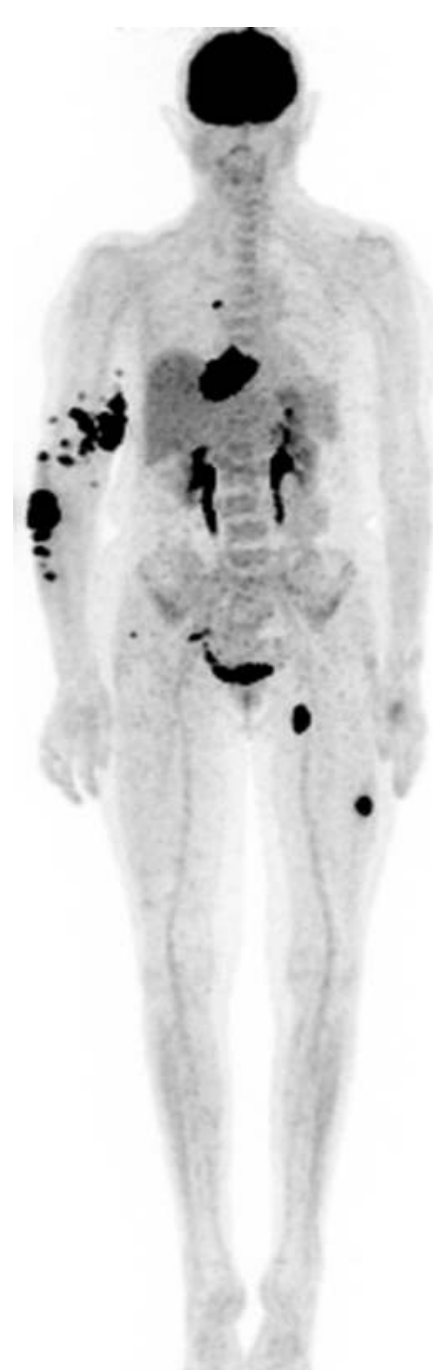

Figure 3: Maximal intensity projection view (MIP) of whole-body 18F-FDG PET. The primary lesion on the medial part of the right breast is visible, and multiple lymphoma lesions identified in the right arm, right hip, and in the lower left leg. 